\title{
Investigation of Different Methods of Noise Reduction for Submerged Marine Propellers and Their Classification
}

\author{
Mohammad A. Feizi Chekab, Parviz Ghadimi*, Seyed Reza Djeddi, Mehdi Soroushan \\ Department of Marine Technology, Amirkabir University of Technology, Tehran, Iran \\ *Corresponding author: pghadimi@aut.ac.ir
}

Received Jnauary 20, 2013; Revised Februay 09, 2013; Accepted April 11, 2013

\begin{abstract}
In this paper, the most prevalent methods of reducing noise from the marine propellers are surveyed and introduced. Subsequently, these methods are analyzed and classified as five different categories. This categorization is conducted from the stand points of technology and cost, the type of noise (cavitation, non-cavitation noise), the method of noise reduction (change in propeller geometry, modification of inlet flow, and confinement of the propeller), frequency of the targeted noise, and simplicity of implementation of the method of noise reduction in the existing vessels. The scientific classification performed in this article would bring about better recognition of and acquaintance with each of the mentioned methods of noise reduction, its limitations in cost and applications, which would in turn help the designers and the decision makers make the right decision under different operational circumstances.
\end{abstract}

Keywords: submerged marine propeller, sound propagation, methods of noise reduction, cavitation noise

\section{Introduction}

One of the most important parameters in designing the marine propulsion systems is the acoustic noise due to the oscillations of pressure in the water surrounding the propeller. Consequently, a lot of efforts have been made to minimize the acoustic noise generated by the propulsion system which accounts for the major source of noise propagation from vessels [1]. In addition to the acoustic pollution that this propagated noise can cause in marine environment $[2,3,4,5,6]$, it can also reduce the pleasure and comfort of marine transportation. On the other hand, propagated noise from the commercial vessels may interfere with the natural echolocation or biosonar systems of whales and dolphins which they use to locate predators and preys and this can confuse them and interfere with their basic biological functions such as feeding and mating [3]. The close resemblance between the propagated noise signals of some vessels and the noise signals of particular species of whales has caused confusion for these mammals in recognizing the ships and has endangered both the whales and the vessels [4]. Last but not least, one of the major methods used in the identification of naval ships and submarines is based on their radiated noise signals.

In the present paper, main tools of propeller noise reduction are presented due to the fact that propellers are largely used as the main propulsion system for major commercial, passenger and naval ships. These methods are investigated and classified from different points of view. These classifications will pave the way for the designers and manufacturers in choosing the best suitable method for their particular operational circumstance.

The history and background of all the reduction tools and the scholars who have done prime research on the development of these methods are presented in the respective sections of each approach. Therefore, the background of the aforementioned methods is not given in the introduction.

There seems to be multitude of tools and ways for reducing the propeller noise which are presented one after another and finally are given inside a general classification that is suggested by the writers of the present work.

\section{Blade Geometry Modification}

Reducing pressure oscillations on the propeller is one of the most effective ways of reducing the radiated noise. Different parameters will affect these oscillations, as follows:
1. Increase in Skew Angle
2. Increase in number of blades
3. Increase in blade area
4. Suitable distribution of blade pitch
5. Trailing edge geometrical modifications
6. Propeller blade finishing fineness

Before moving to next section, it is necessary to explain the geometrical properties and parameters of a conventional marine propeller. Figure 1 shows a general definition of a propeller's blade section.

One of the most important parameters that defines the propeller's geometry is Pitch. This parameter refers to the 
helical progress along a cylindrical surface [7]. This helical progress is presented in Figure 2.

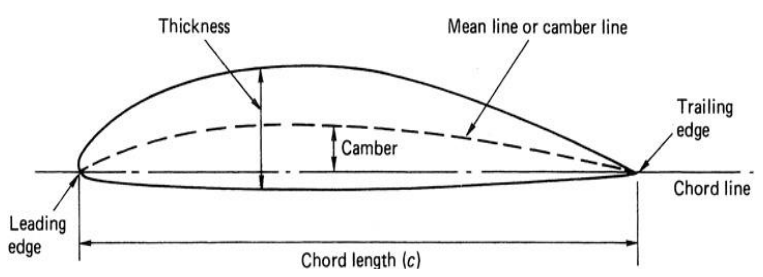

Figure 1. General definition of a propeller's blade section [7]

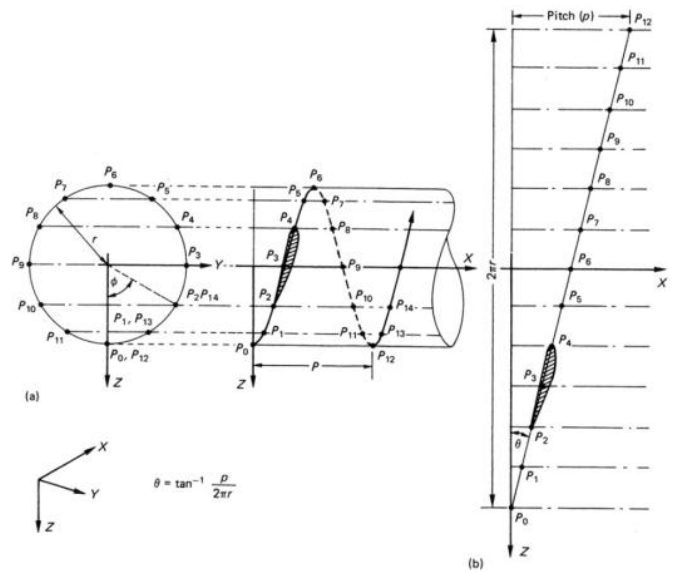

Figure 2. Definition of pitch: (a) helix definition on a cylinder of radius $r$ and (b) development of this helix on the cylinder [7]

Another factor which is often referred to especially in the case of low-noise propellers is the Skew Angle. This parameter which is defined and shown in Figure 3, is the angle between a line that is drawn through the hub center and a point located in the mid-chord position of a particular section and the directional line.

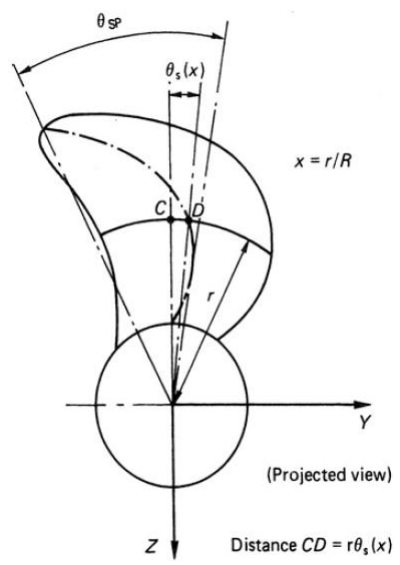

Figure 3. Definition of the propeller's Skew angle [7]

After testing and investigating nine standard propellers in the operational conditions using a $250 \mathrm{hp}$ engine that turns the propeller with the rotational velocity of $1500 \mathrm{rpm}$, it has been shown that an increase in the number of blades from 3 to 5 can lead to approximately $55 \%$ reduction in the propagated noise of the propeller. Also with an increase in skew angle from 12 to 40 degrees, a $48 \%$ average noise reduction can be obtained. Applying both methods at the same time can result in $77 \%$ reduction of the generated noise of the propeller that is measured on the vessel's hull [8].

It is worth mentioning that the cavitation noise plays a significant role in noise signal of a vessel [9]. Therefore, when modifying the propeller geometry, extra care should be given to the effects it might have on the propeller based on both efficiency and noise perspectives. Based on the above, Koushan et al. [10] investigated a systematic method for the optimization of the propeller blades from cavitating and acoustical points of view.

Another experiment in this field was done by Andersen et al. [11] on a Kappel-Type propeller. In this study, two 7 and 8-blades propellers were investigated. It was shown that, with the increase of propeller blades from 7 to 8 , $15 \mathrm{~dB}$ noise level and $80 \%$ thrust oscillations reduction can be achieved. The results of these experiments are given in Figure 4.

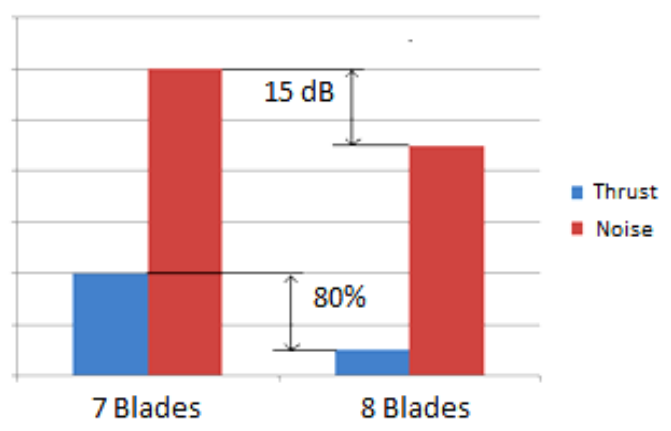

Figure 4. Results of the experiments on Kappel-propellers

Other experimental studies were done by Sharma [12] in India on propeller noise. He used three different types of propellers, i.e. A, B and C.

Propeller A was a TMB-modified NACA-66 while propeller B was a Plano-convex with sharp leading and trailing edges. Specifications of the propellers A and B are given in Table 1.

Table 1. Geometrical Specifications of Propellers A and B

\begin{tabular}{|c|c|c|c|c|}
\hline $\mathrm{r} / \mathrm{r}_{0}$ & $\mathrm{C}_{\mathrm{m}} / \mathrm{c}$ & $\mathrm{t}_{\mathrm{m}} / \mathrm{c}$ & $\mathrm{c} / \mathrm{D}$ & $\mathrm{P} / \mathrm{D}$ \\
\hline 0.25 & 0.037 & 0.253 & 0.174 & 1.092 \\
\hline 0.35 & 0.0388 & 0.192 & 0.193 & 1.093 \\
\hline 0.45 & 0.0372 & 0.146 & 0.212 & 1.092 \\
\hline 0.55 & 0.034 & 0.113 & 0.227 & 1.09 \\
\hline 0.65 & 0.0312 & 0.087 & 0.232 & 1.087 \\
\hline 0.75 & 0.029 & 0.068 & 0.226 & 1.085 \\
\hline 0.85 & 0.027 & 0.052 & 0.202 & 1.084 \\
\hline 0.95 & 0.0247 & 0.045 & 0.139 & 1.083 \\
\hline
\end{tabular}

Propeller C was a Wageningen B4-55 with the specifications given in Table 2 .

Table 2. Geometrical Specifications of Propeller C

\begin{tabular}{|c|c|c|c|}
\hline $\mathrm{r} / \mathrm{r}_{0}$ & $\mathrm{t}_{\mathrm{m}} / \mathrm{c}$ & $\mathrm{c} / \mathrm{D}$ & $\mathrm{P} / \mathrm{D}$ \\
\hline 0.3 & 0.117 & 0.247 & 0.881 \\
\hline 0.4 & 0.101 & 0.273 & 0.934 \\
\hline 0.5 & 0.084 & 0.29 & 0.982 \\
\hline 0.6 & 0.068 & 0.296 & 0.992 \\
\hline 0.7 & 0.057 & 0.289 & 1.004 \\
\hline 0.8 & 0.048 & 0.265 & 0.995 \\
\hline 0.9 & 0.043 & 0.204 & 1.005 \\
\hline
\end{tabular}

Based on the experimental results, the frequency range can be divided into two lower band frequencies ( 0 to $10 \mathrm{kHz}$ ) and higher band frequencies (10 to $100 \mathrm{kHz})$. According to the noise diagrams in two different operational conditions shown on Figures 5 and 6 and based on the hydrodynamic efficiencies, noise of the propellers can be explained. 


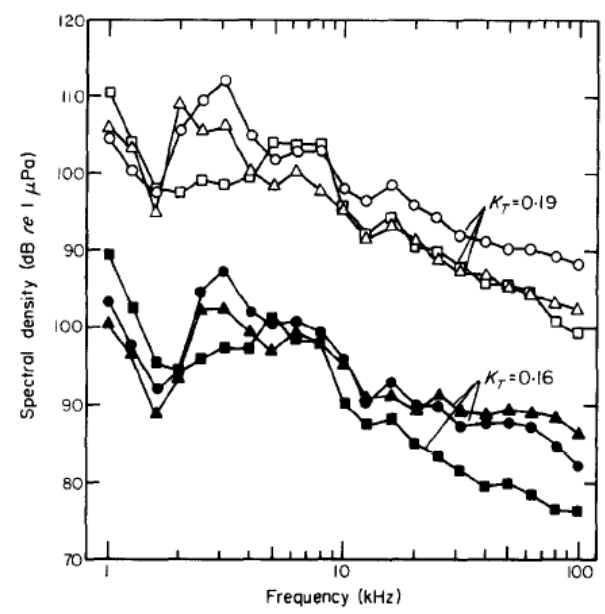

Figure 5. Propeller noise in two different operational conditions: Propeller A (circle), Propeller B (triangle),Propeller C (rectangle)

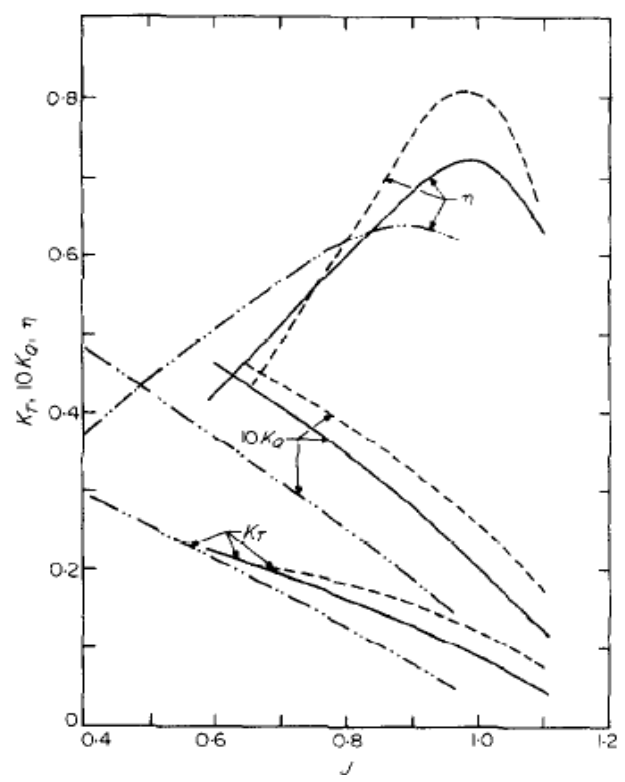

Figure 6. Performance diagram (Efficiency $\eta$, Thrust Coefficient $K_{T}$ and Torque Coefficient $\mathrm{K}_{\mathrm{Q}}$ for Propeller $\mathrm{A}\left({ }_{\ldots}\right)$, Propeller B ( $\left.{ }_{-}\right)$and Propeller C ( _.._ ) vs. Advance Coefficient (J) $J=V / n D$

As seen in Table 3, for the operational condition of $\mathrm{K}_{\mathrm{T}}$ $=0.16$, the efficiency of the Propeller $\mathrm{C}$ is lower than the other two types of propellers but from the hydroacoustic point of view, this propeller seems to be superior to Propellers A and B. It is clear that at the lower frequency range and at the frequency of $5 \mathrm{kHz}$, Propeller $\mathrm{C}$ generates $12 \mathrm{~dB}$ less noise than the other two propellers. At the same time, it is shown that the increase of frequency will bring more reduction in the noise level of Propeller C. Propeller A and B have similar characteristics, but overall, Propeller $\mathrm{B}$ generates less noise. From the efficiency perspective and at the operational condition of $\mathrm{K}_{\mathrm{T}}=0.19$, Propeller $\mathrm{C}$ stands in the middle and like before, it generates the lowest noise. A similar noise will be radiated from Propeller B at its highest efficiency level in high frequency range and Propeller A with its lowest efficiency will generate the most highest noise.

Table 3. Efficiencies of Propellers A, B and C in two different

\begin{tabular}{|c|c|c|c|}
\hline \multirow{2}{*}{$\mathrm{KT}$} & \multicolumn{3}{|c|}{ Efficiency } \\
\cline { 2 - 4 } & $\mathrm{A}(\%)$ & $\mathrm{B}(\%)$ & $\mathrm{C}(\%)$ \\
\hline 0.16 & 59.7 & 72.5 & 57.6 \\
\hline 0.19 & 51.6 & 57.2 & 53 \\
\hline
\end{tabular}

In order to solve this problem, Sharma [12] modified the blades of Propellers A and B by drilling 300 holes with the diameters of $0.3 \mathrm{~mm}$. With this modification, a $50 \%$ reduction was achieved in propeller tip vortex which consequently resulted in a reduction in the propeller noise.

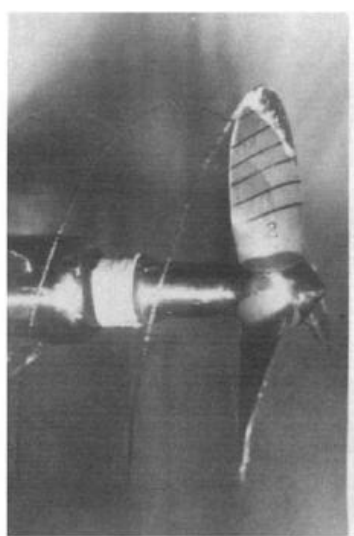

(a)

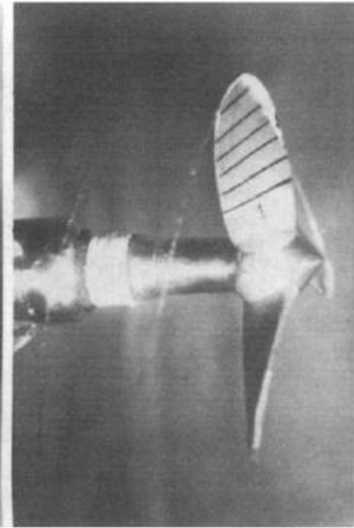

(b)
Figure 7. Propeller A in its original form (Left) and modified form (Right)

As evidenced in Figure 7, Sharma's modifications led to a decrease in cavitation and therefore a reduction of noise level.

Propeller's trailing edge (TE) geometry and its boundary layer play a significant role in the radiated noise of the propeller. Wang et al. [13] have done an extensive and precise study in 2006 on the effects of the propeller's trailing edge on the radiated noise. In this study, using numerical simulations, optimization of the trailing edge geometry was investigated. As a result of this optimization study, blades' trailing edge vortices and subsequently their generated noises were reduced especially in the case of low-frequency noise signatures. Results of this study are shown in Figure 8.

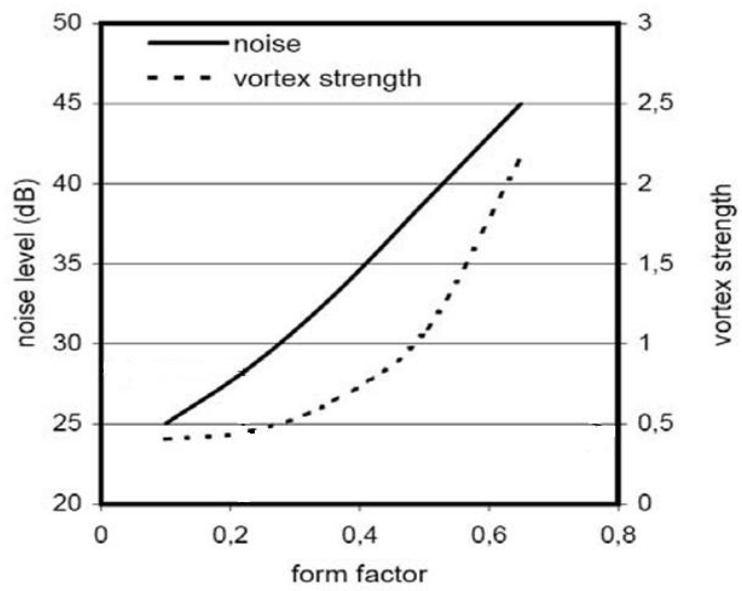

Figure 8. Trailing edge noise and vortex strength [circulation $\left(\mathrm{m}^{2} / \mathrm{s}\right.$ ] $\left(\Gamma=\int \omega d a\right)$ vs. trailing edge aspect ratio or form factor

It needs to be added that this study was actually a continuation of the Blake's [14] experiments in 1986 which was done on the effects of the local variations of the trailing edge aspect ratio in 2-dimensional configurations. In his studies, Blake varied the trailing edge from a very thick form to a very thin or sharp form. As observed in Figure 8, a sharper trailing edge will result in less noise radiation. 


\section{Using Contracted and Loaded Tip (CLT)}

Another technique of noise reduction is the confinement of propeller's tip with a plane. An example of this method is shown in Figure 9. Confining the propeller's trailing edge will result in less circulation around the blade's tip and therefore the propeller will be put in heavier conditions. With this tool, diameter of the optimized propeller will be reduced and consequently the probability of cavitation occurrence will be decreased.

This system was first introduced by SISTEMAR Company and in an article by Gomez and Gonzalez [15] in 1995, the physical justification of this method was presented while the CLT theory was proven using the formulation and governing equations of the momentum theory.

In 2005, SISTEMAR performed experiments on two similar 164,000 tonnes bulk carrier ships and installed a new CLT propeller on one of them. Results showed that the ship with old type propeller had $12 \%$ more fuel consumption.

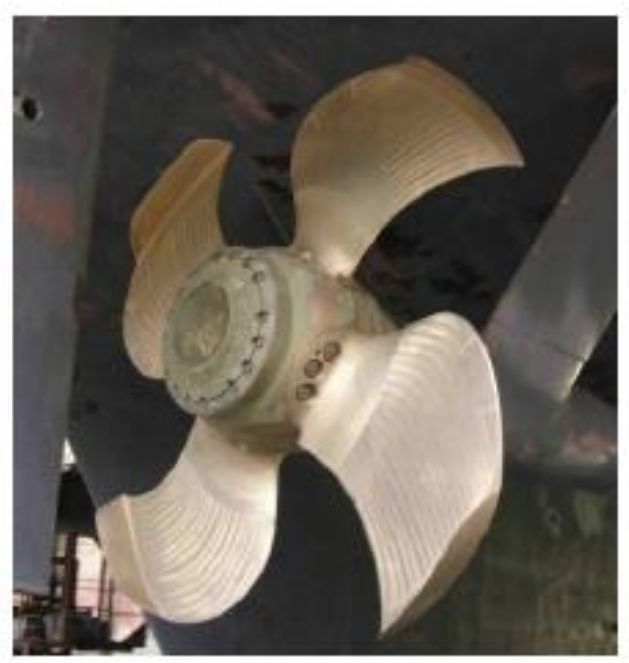

Figure 9. CLT propeller installed on a bulk carrier

Also, the same system was installed on a passenger ship with twin screws propulsion system which used to suffer from vibrations problem [16]. This passenger ship had a length of 157 meters with 15,327 tonnes displacement. After the installation, interesting results were obtained. At speeds of 21 to 24 knots, $11 \%$ and at the speed of 15 knots, $30 \%$ reduction of engine power consumption was achieved. Additionally, propellers' pressure spectrum was reduced.

This type of propeller has many advantages but extra care should be given to its design.

\section{Kappel Propellers}

Kappel propellers were first introduced by Andersen et al. [11] in 2000. In these types of propellers, the trailing edge is curved toward the suction region (back-face) and therefore in addition to reducing cavitation and respective cavitation noise, a $4 \%$ increase in the propeller's hydrodynamic efficiency can be obtained [16]. A typical Kappel propeller is shown on Figure 10.

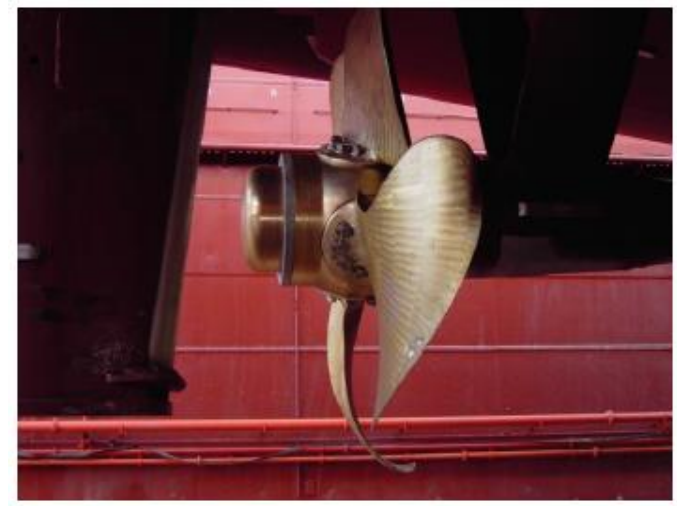

Figure 10. Kappel Propeller installed on a ship

\section{Propeller Hub Modification}

One of the propeller parts that play a significant role in introducing the pressure gradients and therefore the irregularities in the pressure distribution is the propeller's hub section. Effects of the blades are less in this section and therefore the velocity and pressure of water in this region is much lower than that of propeller's mid-regions. As a result, the hub section is exposed to cavitation and pressure oscillations. Consequently, this section should be given extra attention from cavitating and non-cavitating noise perspectives.

One of the methods that can be used in order to reduce the radiated noise in this section is the implementation of Propeller Boss Cap Fins (PBCF) on the propeller's hub as shown in Figure 11. With this tool, in addition to the decrease in fluid's circulation on the hub that will be achieved, hub cavitation can also be reduced [16].

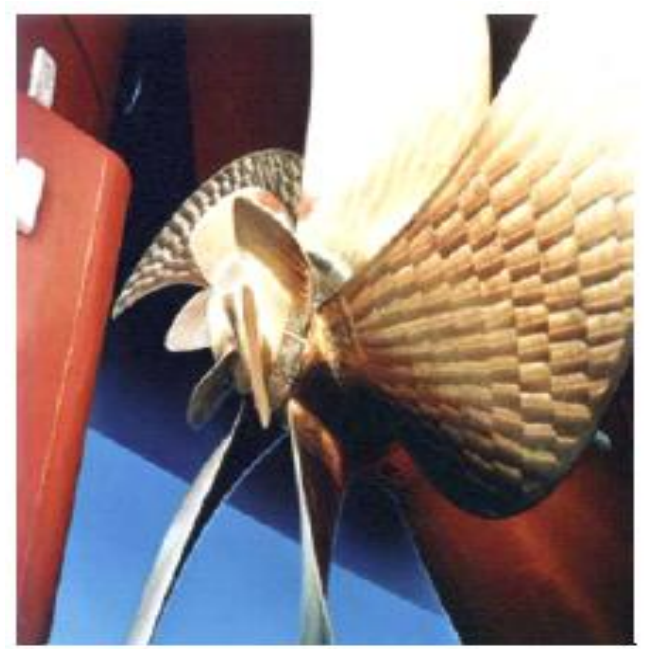

Figure 11. An example of using PBCF system on a marine propeller

This system has many advantages such as:

1. More efficiency (approx. 7\%)

2. Cavitation tunnel test proved that sound pressure level (SPL) will be reduced by 3 to $6 \mathrm{~dB}$ in frequencies higher than $1 \mathrm{kHz}$

3. The ship does not need to be dry-docked for the installations of these fins and they can be fitted to propeller while the ship is still afloat

4. Use of PBCF will cancel hub vortex or hub cavitation (as shown on Figure 12) and therefore one of the major noise sources is attenuated 

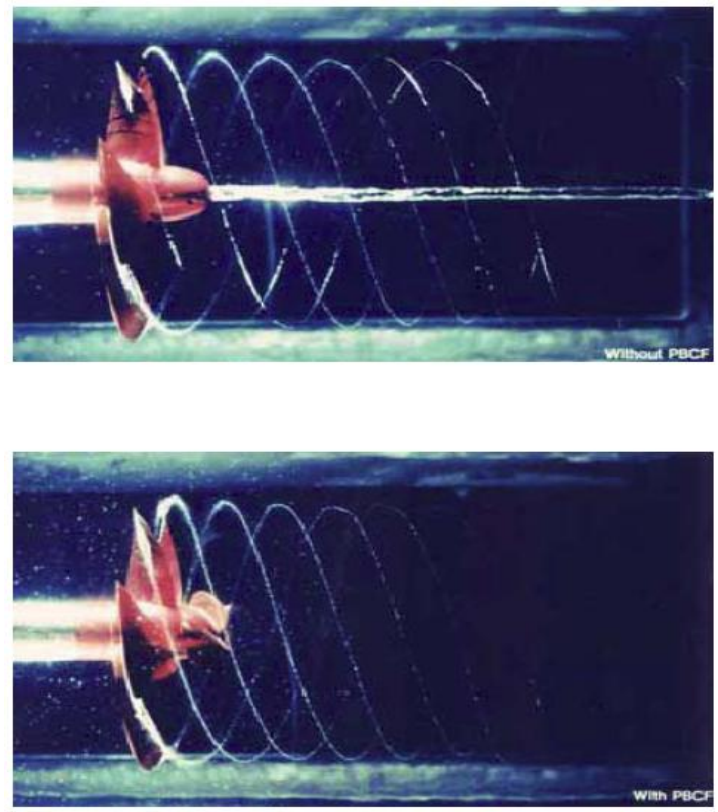

Figure 12. Ordinary propeller with hub cavitation (top) and the Propeller equipped with PBCF that cancels hub cavitation (bottom).

Another cavitation and hub noise reduction method is the use of multiple hydrofoils on the hub which is called Propeller Cap Turbine (PCT). Similar to PBCF, PCT will cause less energy consumption while weighting around 1 to $2 \%$ of the total propeller's weight. A schematic of this system is shown [16] in Figure 13.

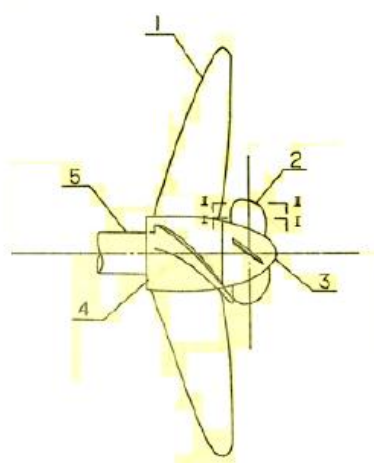

Figure 13. A schematic of PCT system

\section{Wake Inflow Modification}

Although the propeller noise is due to the propeller rotation and the consequent pressure distribution around it, propeller's wake inflow plays a significant role in the propeller performance and the probability of cavitation [17]. On the other hand, perturbation and fluctuations of free-surface can also affect the propeller's performance [18].

Therefore, in addition to the reduction methods mentioned in the previous sections, other methods are developed based on the modification of wake inflow. With the implementation of special elements that will be installed on the hull, a uniform inflow will be provided for the propeller which will abate propeller cavitation, propeller thrust oscillations and propeller vibrations. In addition to the increase in the propeller's efficiency, these methods will also decrease the radiated noise level.
An example of these elements is the Schneekluth duct. The idea behind this element is developed in order to change the propeller's upstream flow which will result in less generated noise that will be radiated from the propeller's blade trailing edge. Consequently, the sound pressure level (SPL) will be decreased. However, there is not sufficient information about the effectiveness of this method in reducing the generated noise in all existing vessels. The Schneekluth duct will be installed on the hull of the ship and with the help of this element in addition to $12 \%$ decrease in fuel consumption, $50 \%$ less vibration will also be achieved. For example, the system has been installed on a 2500 tonnes Container ship with 22 knots speed. As a result of this modification, the annual fuel consumption has been decreased by up to 1200 tonnes. An example of this duct which is installed on a ship is shown [16] in Figure 14.

Another element in this group of methods is the Mewis duct (shown in Figure 15). This element is placed just before the propeller and has been designed by Becker Marine Systems. Mewis duct will also bring better and more uniformed wake inflow for the propeller.

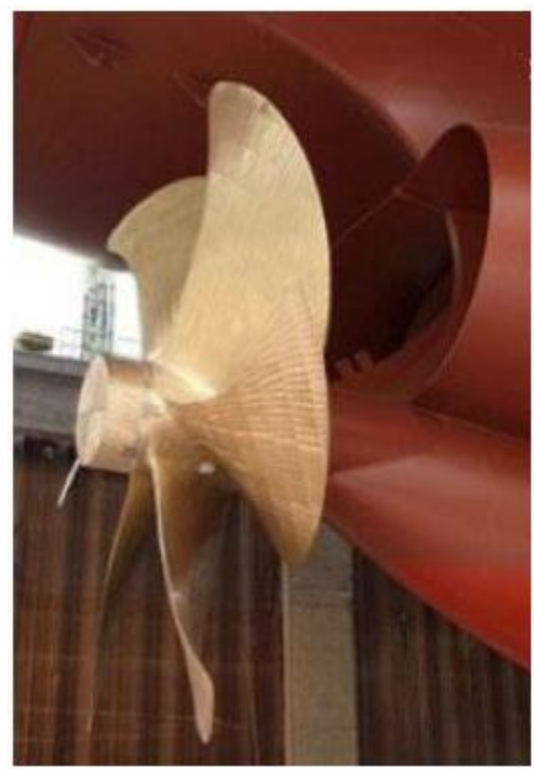

Figure 14. Schneekluth duct installed on a ship

Installation of this system on a VLCC ship with the length of $318 \mathrm{~m}$, breadth of $60 \mathrm{~m}$, water draft of $20 \mathrm{~m}, 16$ knots speed and 22,000 KW engine power has led to $5 \%$ less fuel consumption. Additionally, results show that with the modification of wake inflow, the efficiency of the propulsion system has been increased and propeller's cavitation and vibrations have been reduced [16].

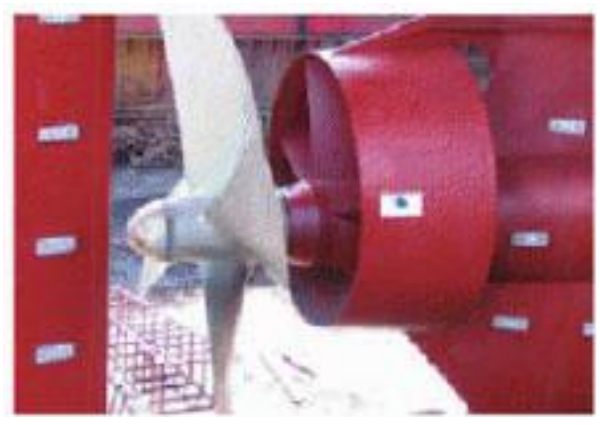

Figure 15. Mewis duct system 
Another method of modifying the wake inflow is called Simplified Compensative Nozzle or SCN. This tool is installed on both sides of the hull in the aft section as shown in Figure 16. The system in resemblance with the two latter methods will result in uniformity of the wake inflow in the stern region and will therefore reduce the generated noise due to the propeller's inflow oscillations. In addition to the advantages mentioned above, SCN system can be built a lot easier in compare to other inflow modification tools that were previously introduced [16].

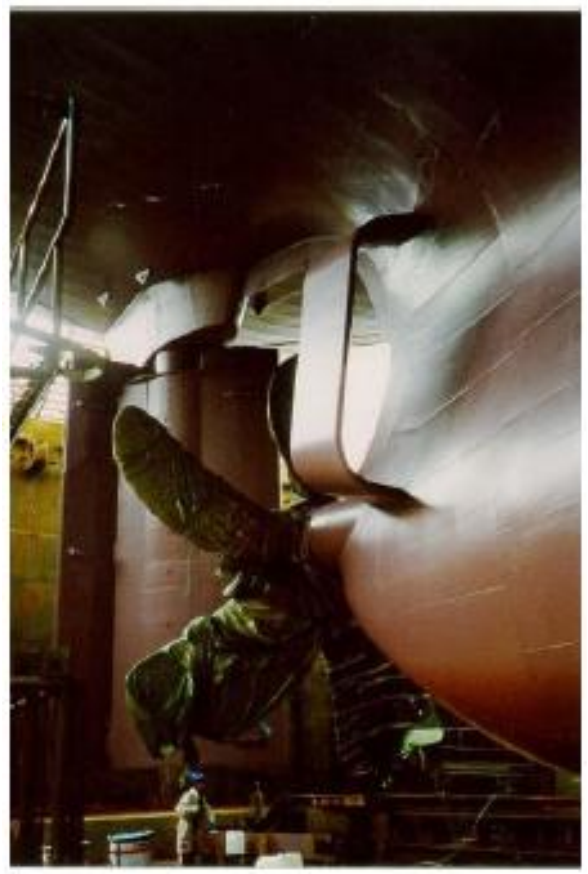

Figure 16. Simplified Compensative Nozzle or SCN system

\section{Propeller's Confinement: Different Types of Ducts}

Another family of methods that can be used in order to reduce the radiated noise from the propeller propulsion system both in marine and aviation applications is the use of ducts which surround the propeller. Generally, in aviation industries, two types of conical and cylindrical ducts are mainly used. With the implementation of these ducts, in addition to the propeller's efficiency increase, reduction in radial propagated noise will also be achieved. A practical way of improving acoustical behavior of these ducts is to use effective sound absorbers inside them.

In marine industries, ducted propellers are widely used. These ducted propellers are often called Kort Nozzle systems. Nowadays, different types of ducts are introduced with the most common types depicted in Figure 17. In general, there are two types of ducts in marine applications; accelerating and decelerating. The type that is shown in Figure 17-a is called Wageningen 19A which is among the most widely used ducts due to its high efficiency in forward thrust. This type of duct is suitable for vessels that do not require astern or backward sailing and cannot be effectively used for towing tugboats. For this reason, another type of duct (Figure 17-b) is introduced. For this type of duct, the astern sailing has more efficiency and on the other hand the efficiency for the forward sailing will be reduced. In the past two decades, investigations have been carried out for the improvements of efficiency in astern sailing for the ducted propellers. As a result of these investigations a new type of ducts (Figure 17-c) are introduced. In addition to the hydrodynamic section design in accordance with the forward sailing efficiency, these ducts take advantage of particular holes which are placed at the back of the ducts. In times of backward sailing, these holes let the water pass through and therefore the ducts will have the same performance as the first type of ducts [7].

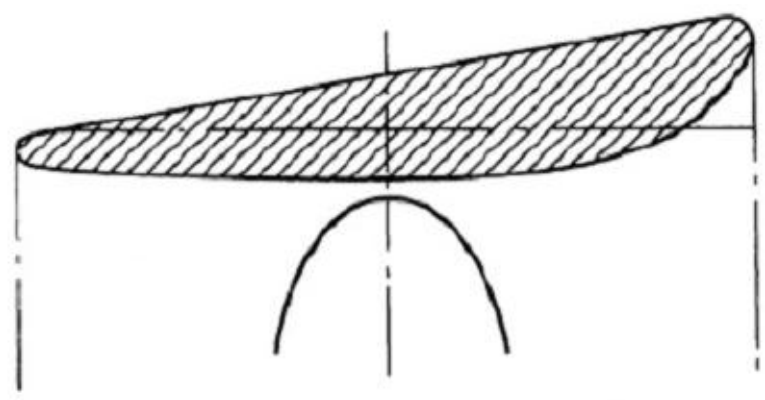

[a]

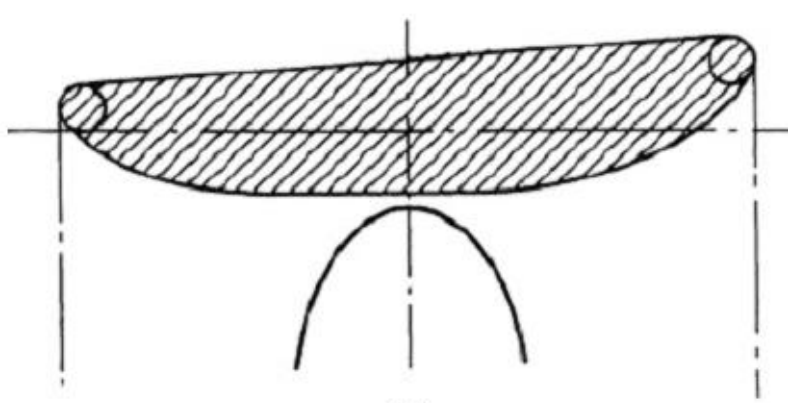

(b)

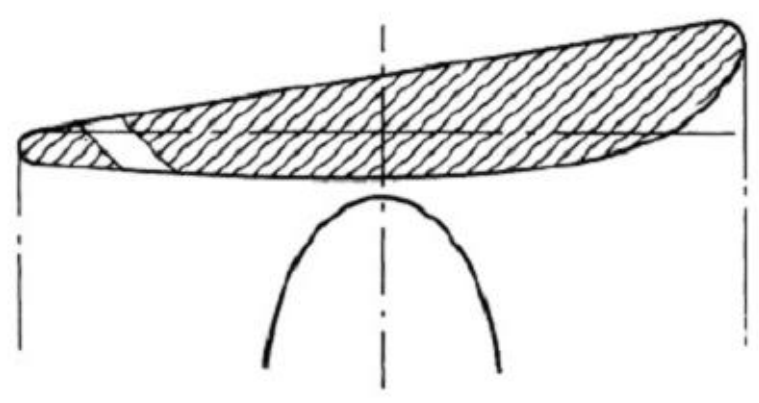

(c)

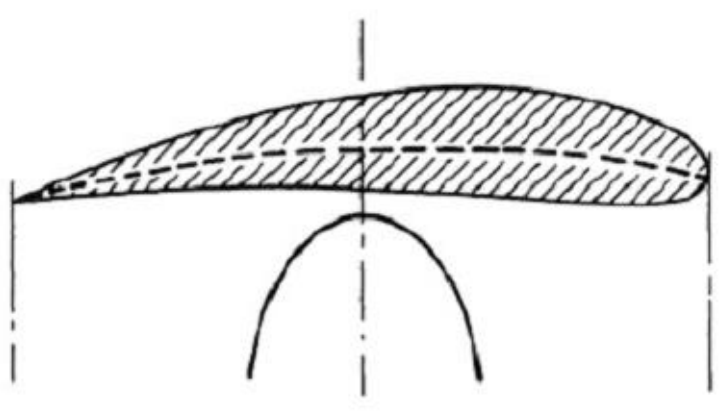

(d)

Figure 17. Different types of ducts used in marine industries for noise reduction applications 
For the sake of cavitation control and noise reduction, decelerating ducts are often used. This type of ducts which is illustrated in Figure 17-d improves the cavitation conditions by decreasing the propeller's inflow speed. These ducts are mainly used in naval ships, submarines and torpedoes.

In 2010, Haimov et al. [19] conducted some experiments on the performance and design methods of ducted propellers. Also, the studies of Seol et al. [20] in 2002 are among the most influential research works in this field. In their work [20], using a finite element method solver, they solved the potential flow around the propeller which later used as the input of the Ffowcs WilliamsHawkings (FWH) formulation. The FWH equations are solved using a boundary element solver and the far-field noise has been calculated for a typical propeller geometry. Later, using the presented method, the acoustical analysis has been performed on a Kappel Series propeller.

In the present section, different methods of reducing and controlling propeller noise were introduced. For a better overview of these methods and more practical applications, different classifications are presented in the following section.

\section{Classification of Noise Reduction Methods for Marine Propellers}

For more efficient application of the noise reduction methods in the design of marine propellers, various classifications based on different perspectives are required. An example of these classifications was presented in the proceedings of the National Oceanic and Atmospheric Administration (NOOA) International Symposium in 2007 for large commercial ships [3], while this classification was mainly based on the implementation cost for each method.

Noise reduction methods can be classified in different categories. One of the suitable classifications is based on the type of the reduced noise, i.e.:

1. Cavitation Noise Reduction

2. Non-Cavitation Noise Reduction

3. Radiated Noise Reduction

Another important classification is based on the modification that is required for the implementation of the reduction method:

1. Propeller Wake Inflow Modification

2. Propeller Geometry Modification

3. Propeller Isolation

A combination of these two classifications are illustrated in Figure 18.

In addition to the classifications presented above, noise reduction methods can also be classified in terms of cost and required technology. If a score of 0 to 1 is assigned to each method in such a way that one (1) refers to the highest and zero (0) refers to the lowest level of cost/technology requirement, the diagram presented in Figure 19 will be achieved.

The vertical axis depicts the weighted parameters which account for each of the modification methods. The respective weights are estimated using the international figures for propulsion system installation/modification cost.
As evidenced in Figure 19, modifications in the propeller geometry is actually the most expensive method in sense of cost and technology with propeller isolation and wake inflow modification methods coming next. Propeller design is truly an intricate and expensive procedure and a slight fault in this phase can lead to less predicted efficiency in comparison to the standard calculations.

Noise reduction methods in sense of controlling particular frequency ranges are proved to be important and lead to a new classification of the methods. This classification includes low frequency and high frequency noise reduction methods which are depicted in Figure 20.

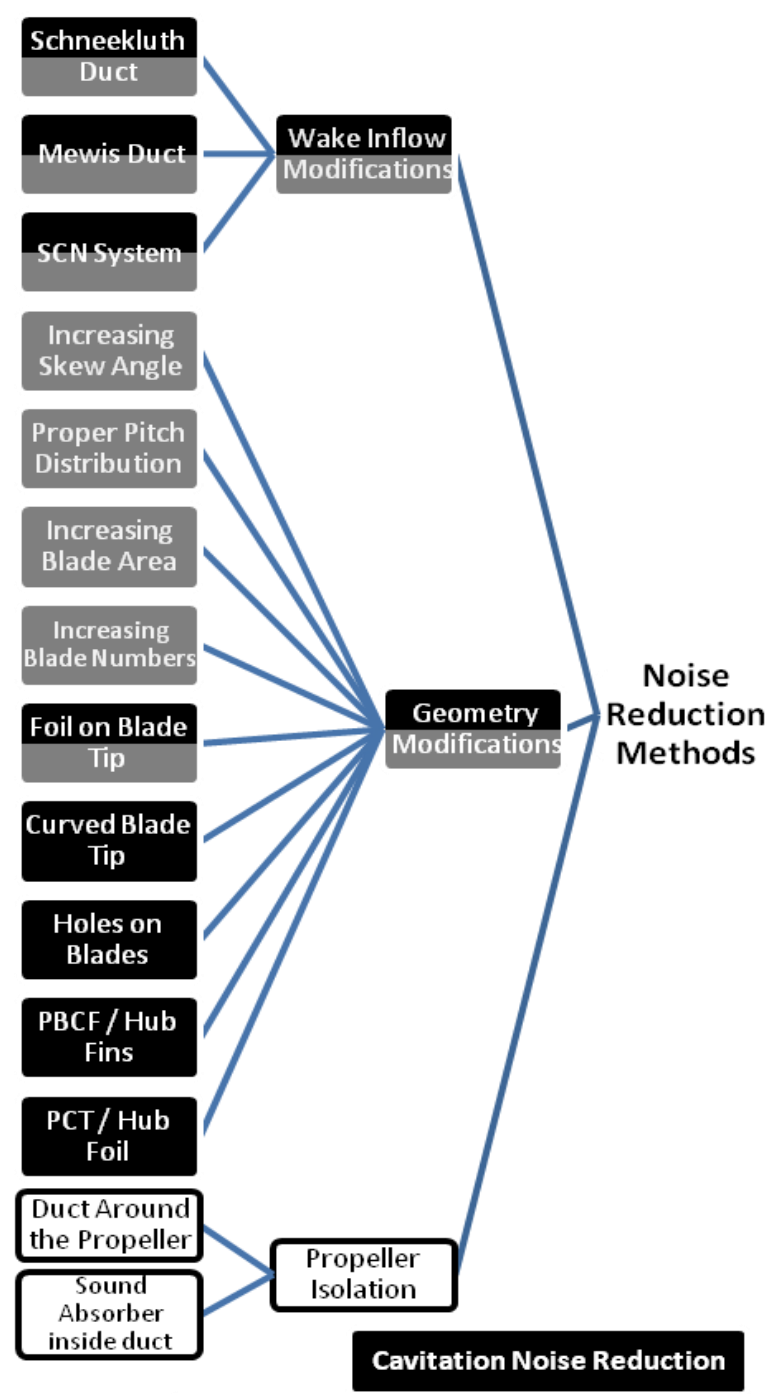

Non-Cavitation Noise Reduction

Radiated Noise Reduction

Figure 18. Noise reduction methods and their classification

High frequency noise reduction methods, generally attenuate or abate cavitation while the wake inflow or flow around the propeller are modified in low frequency noise reduction methods and the radiation of noise in this group of methods is controlled.

Based on these classifications, choosing a suitable reduction method would become easier when decisions are going to be made about the propulsion system of a vessel. 


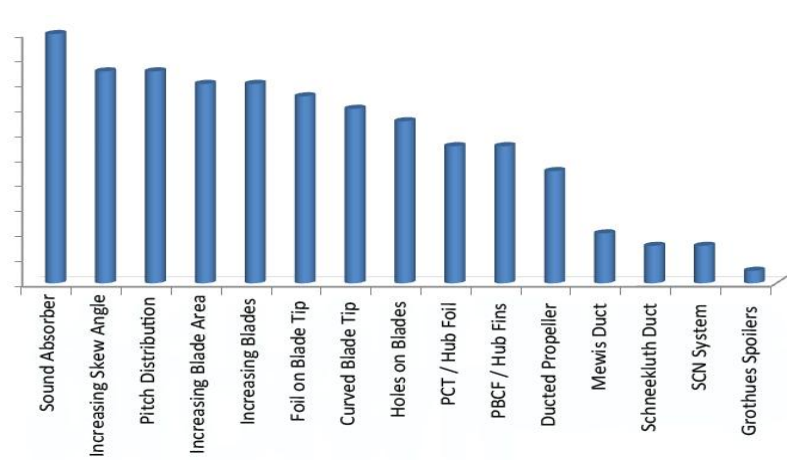

Figure 19. Noise Reduction Methods assortment

Another approach for classifying the noise reduction tools is based on the simplicity of the method's application on the existing vessels. In this classification, hydroacoustic optimization of propeller would be among the hardest methods while using ducts proves to be the easiest one. Figure 21 shows this system of classification.

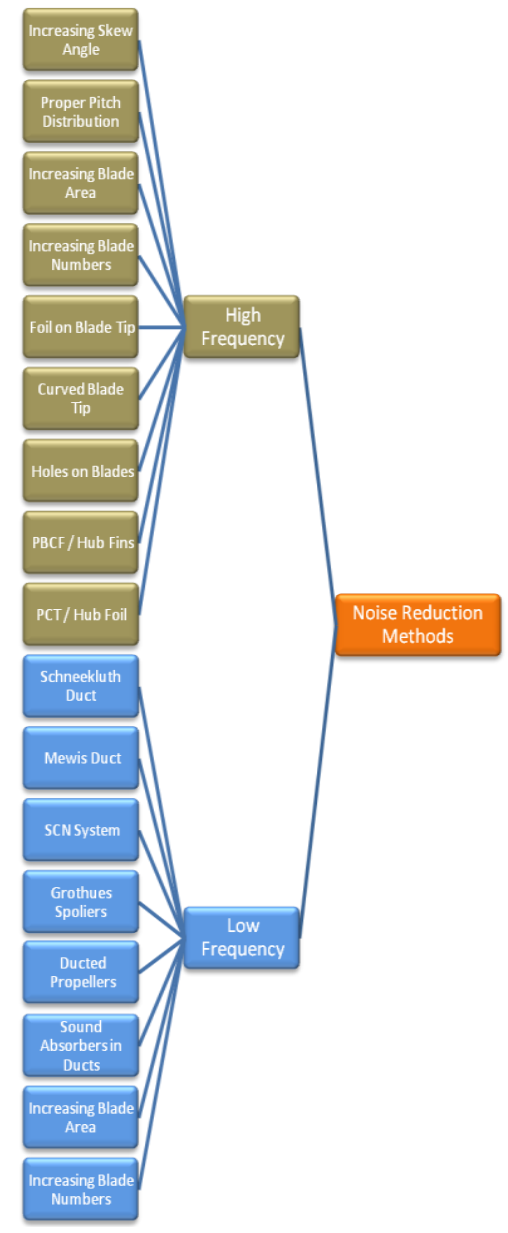

Figure 20. Classification of different noise reduction methods based on the frequency range of the attenuated noise

\section{Conclusions}

In the present paper, principal methods of marine propeller noise reduction are presented and studied. These methods are then classified in five different categories which bring about better acquaintance with each of the methods, their limitations in cost and applications, which will help the designers make the right decision under different operational circumstances.
Studying different classifications proves that using ducts (in the aft region or around the propeller) is the easiest, most operative and most cost effective method in compare to the method of redesigning propellers for the existing vessels. Therefore, in order to control the radiated noise of the existing vessels, installation of ducts would be the first priority. This decision is due to the fact that by using ducts, radiated noise will be reduced, propeller's efficiency will be improved and the cavitation shedding will be delayed. Furthermore, using ducts can be the best and most effective choice for the vessels that are currently built and in operation. As the second priority, equalizing wake inflow with the help of aft ducts are presented which leads to reduced cavitation noise and improved propeller efficiency.

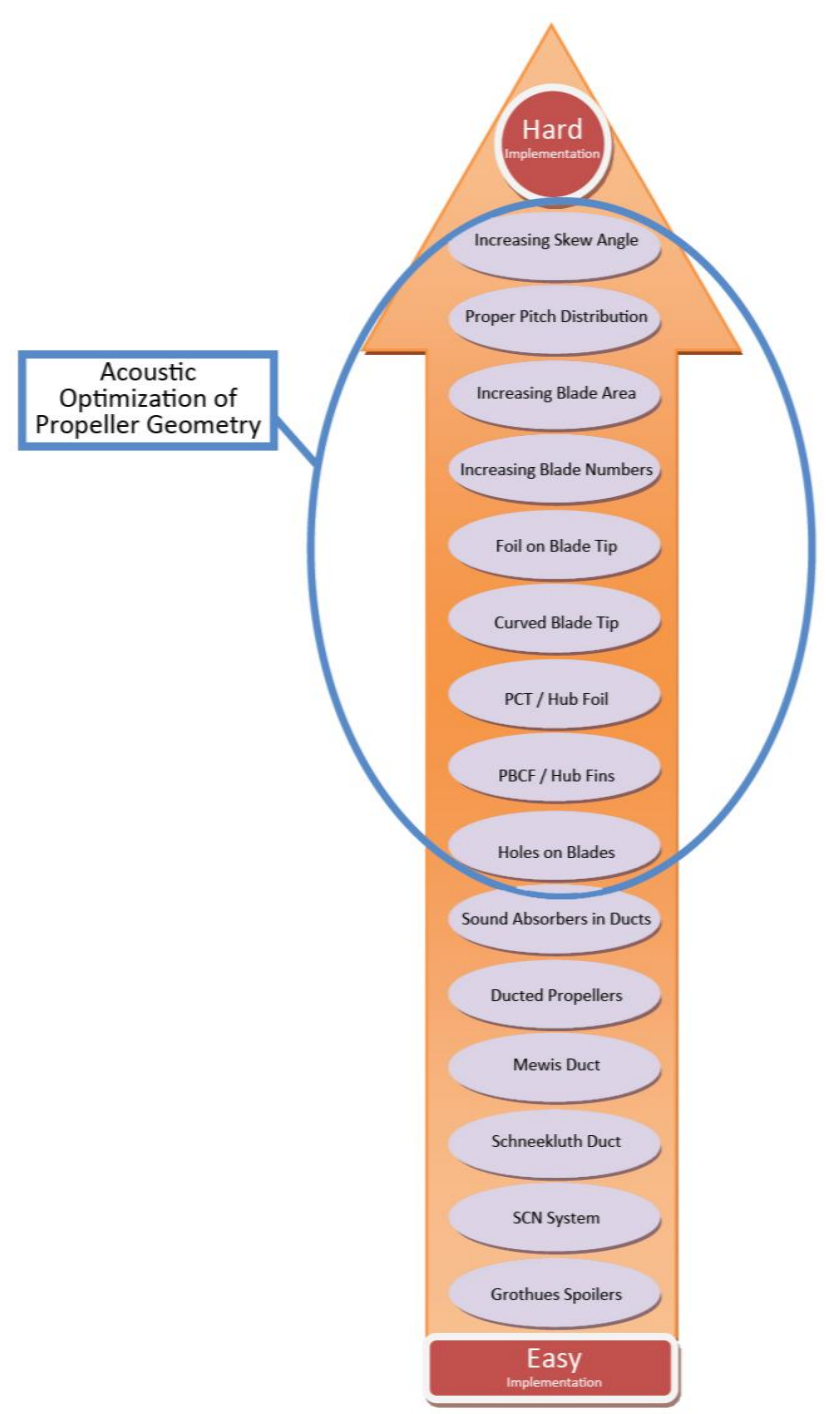

Figure 21. Classification of noise reduction methods based on the simplicity of application

\section{Nomenclature}

$\begin{array}{ll}r & \text { radius } \\ r_{0} & \text { propeller's blade radius } \\ C_{m} & \text { maximum camber length } \\ c & \text { chord length } \\ t_{m} & \text { maximum blade thickness } \\ D & \text { propeller diameter } \\ P & \text { propeller pitch }\end{array}$


$\theta_{S P}$ propeller skew angle

$K_{T} \quad$ thrust coefficient

$K_{Q}$ torque coefficient

$\eta \quad$ propeller efficiency

$J \quad$ advance coefficient

$V \quad$ ship speed

$n \quad$ propeller revolutions per second

$d B \quad$ decibel $\left(d B=20 \log \frac{P}{P_{\text {ref }}}\right)$

$P \quad$ sound pressure $(\mathrm{Pa})$

$P_{\text {ref }}$ reference sound pressure level (in water

$P_{r e f}=1 \mu \mathrm{Pa}$, in air $\left.P_{r e f}=20 \mu \mathrm{Pa}\right)$

$\Gamma \quad$ circulation (vortex strength) $\Gamma=\int \omega d a$

$S P L$ sound pressure level $(\mathrm{dB})$

\section{References}

[1] Ross, D., Ship sources of ambient noise, IEEE J. Oceanic. Eng. 30, 257-261, 2005.

[2] Southall, B.L., Shipping Noise and Marine Mammals: a Forum for Science, Management, and Technology, Final Report of the National Oceanic and Atmospheric Administration (NOAA) International Symposium 2005, U.S.A. NOAA Fisheries, Arlington, Virginia, May 18-19, 2004, 40 pp.

[3] Southall, B.L., Potential Application of Quieting Technology on Large Commercial Vessels, Final Report of the National Oceanic and Atmospheric Administration (NOAA) International Symposium. U.S. NOAA Fisheries, Silver Spring, Maryland, May 1-2, 2007.

[4] Marine mammals and noise a sound approach to research and management, A report to Congress from the Marine Mammal Commission, USA, March 2007.

[5] Shipping noise and marine mammals, A background paper produced by participants of the International Workshop on Shipping Noise and Marine Mammals, Okeanos Foundation for the Sea, Hamburg, Germany, 21st-24th April 2008.

[6] Underwater Radiated Noise of Ocean-Going Merchant Ships, A background paper produced by participants of the International
Workshop on Shipping Noise and Marine Mammals, Okeanos Foundation for the Sea, Hamburg, Germany, 21st-24th April 2008.

[7] John Carlton, Marine propellers and propulsion, Elsevier, Second edition, 2007.

[8] NVT Institute, Secrets of marine acoustical success cruising comfort., undated.

[9] Matusiak, J., Pressure and noise induced by a cavitating marine screw propeller, Doctor of Philosophy thesis, VTT Publications 87, 1992.

[10] Koushan, K, Halstensen, SO, and Sandtorv, LG, 2000, Systematic investigation of blade design influence on cavitation performance and on induced pressure pulses, Proceedings of NCT 50, International Conference on Propeller Cavitation, 3-5 April 2000, University of Newcastle, UK.

[11] Andersen, P., Kappel, J.J., Spangenberg, E., Aspects of propeller developments for a submarine, First International Symposium on Marine Propulsors, smp'09, Norway, June 2009.

[12] Sharma, S. D., Mani, K., Arakeri, V. H., Cavitation Noise Studies on Marine Propellers, Journal of Sound and Vibration Vol. 138, 255-283, 1990.

[13] Wang, M., Marsden, A.L., Moin, P., Computation and Control of Trailing-Edge Noise, Proceedings of the 26th Symposium on Naval Hydrodynamics, Rome, 2006, Italy.

[14] Blake, W. K., Mechanics of flow-Induced Sound and Vibration, Vol. II, Complex Flow-Structure Interactions, Applied Mathematics and Mechanics, 1986, Academic Press.

[15] Gomez, G.P., Gonzalez-Adalid, J., Tip loaded propellers (CLT): Justification of their advantages over conventional propellers using momentum theory, International Shipbuilding Progress, 42 no 429, pp 5-60, 1995.

[16] Renilson Marine Consulting Pty Ltd., Reducing underwater noise pollution from large commercial vessels, Commissioned by The International Fund for Animal Welfare, March 2009.

[17] Ball, W., An experimental investigation into the influence of wake on cavitation, Transactions of the Royal Institution of Naval Architects, Volume 131, pp 73-82, 1989.

[18] Arndt, REA, Holl, JW, Bohn, JC, Bechtel, T, Influence of surface irregularities on cavitation performance, Journal of Ship Research, Volume 23, Number 3, September 1979, pp 157-170, 1979.

[19] Haimov, H., Bobo, M. J., Vicario, J., del Corral, J., Ducted propellers: a solution for better propulsion of ships. calculations and practice, First International Symposium on Fishing Vessel Energy Efficiency, E-Fishing, Vigo, Spain, May 2010.

[20] Seol , H., Jung , B., Suh , J.-C., Lee S., Prediction of noncavitating underwater propeller noise, Journal of Sound and Vibration, Vol. 257, 131-156, 2002. 